Meta

Journal des traducteurs

Translators' Journal

\title{
La métaphore traduisible
}

\section{Moshe Azar}

Volume 34, numéro 4, décembre 1989

URI : https://id.erudit.org/iderudit/001928ar

DOI : https://doi.org/10.7202/001928ar

Aller au sommaire du numéro

Éditeur(s)

Les Presses de l'Université de Montréal

ISSN

0026-0452 (imprimé)

1492-1421 (numérique)

Découvrir la revue

Citer cette note

Azar, M. (1989). La métaphore traduisible. Meta, 34(4), 794-796.

https://doi.org/10.7202/001928ar

Ce document est protégé par la loi sur le droit d'auteur. L’utilisation des services d'Érudit (y compris la reproduction) est assujettie à sa politique d'utilisation que vous pouvez consulter en ligne.

https://apropos.erudit.org/fr/usagers/politique-dutilisation/
Cet article est diffusé et préservé par Érudit.

Érudit est un consortium interuniversitaire sans but lucratif composé de l'Université de Montréal, l'Université Laval et l'Université du Québec à Montréal. Il a pour mission la promotion et la valorisation de la recherche. https://www.erudit.org/fr/ 


\section{BLOC-NOTES}

\section{LA MÉTAPHORE TRADUISIBLE}

Le but de cet article est d'examiner le type de métaphore qui se prête le mieux à la traduction littérale.

Le Guern, dans un article très intéressant, intitulé «Métaphore et argumentation» (1981), écrit à ce sujet des choses très courtes mais aussi très claires. Mais avant que nous citions les quelques lignes de son article, il nous faut revenir à son livre Sémantique de la métaphore et de la métonymie (1973), où il fait la distinction entre métaphore et symbole (pp. 39-47). Quand Péguy écrit que «la Foi est un grand arbre» il crée un symbole, car

au signifiant «arbre» correspond un signifié qui est le concept de l'arbre ou plus exactement la représentation mentale d'un arbre.

Dans l'expression symbolique, ce signifié devient à son tour le signifiant d'un autre signifié, qui sera ici la représentation ou le concept de la foi. On pourra donc dire qu'il y a symbole quand le signifié normal du mot employé fonctionne comme signifiant d'un second signifié qui sera l'objet symbolisé. En toute rigueur, ce n'est pas le mot «arbre» qui est le symbole, mais son signifié, la représentation de l'arbre.

D'après Le Guern (1981: 66), le symbole n'est que le quatrième type de métaphore mentionné par Aristote, e'est-à-dire, la métaphore en vertu d'une relation de proportionnalité. En revenant à l'exemple de Péguy, nous pouvons dire que l'arbre est à la foi ce que le bourgeon est à l'espérance.

La métaphore propre, au contraire de la métaphore-symbole, est en vertu d'un transfert d'espèce à espèce comme dans «vous êtes mon lion». Ici, le substantif «lion» ne correspond pas au signifié habituel de ce mot, le signifié du mot «lion» est ce qu'il y a de commun aux deux représentations, celle du lion et celle de la personne qualifiée. Il faut qu'il y ait un attribut dominant qui soit le trait de similarité servant de fondement à l'établissement du rapport métaphorique. Selon Le Guern l'attribut dominant, par exemple «courage», fait partie des sèmes du mot «lion».

Nous pourrons passer maintenant à ce que dit Le Guern au sujet de la traduction de la métaphore et du symbole :

Meta, XXXN, 4, 1989
Pour qu'une métaphore soit possible, il faut que l'attribut commun appartienne au signifié du terme métaphorique. Le symbole, en revanche, peut se fonder sur n'importe quelle analogie entre traits de substance : il n'est nullement nécessaire que ces traits de substance aient été retenus comme sèmes pour la langue dans laquelle on s'exprime. C'est pour cette raison que le symbole dépend bien moins que la métaphore de la structure sémantique de la langue: le symbole se traduit normalement quand on passe d'une langue à une autre, alors que Ia métaphore n'est pas traduisible, à moins d'un heureux hasard. Alors que les métaphores sont le meilleur moyen d'atteindre les sèmes d'une langue, les symboles n'apprennent rien sur la langue elle-même (1981: 67).

J'adhère complètement à l'opinion selon laquelle le symbole, tel qu'il a été défini par $\mathrm{Le}$ Guern, est en principe traduisible. De même que la similitude, il ne peut lui non plus être saisi par le destinataire que s'il se représente les mots dans leur sens propre. La seule différence entre symbole et similitude est que la dernière n'impose pas un transfert de signification et qu'aucune incompatibilité sémantique n'est perçue, et nous avons vu que ce transfert de signification ne s'établit pas sur le plan sémique, mais symbolique : le signifié du mot métaphorique sert de signifiant d'un second signifié.

En ce qui concerne la métaphore propre, je ne crois pas que nous devions accepter la conclusion qu'elle n'est jamais traduisible. Le Guern (1981: 67) lui-même admet qu'il est parfois très difficile de savoir si l'on a affaire à une métaphore ou à un symbole. Ces difficultés proviennent du côté du sémanticien, qui a tendance à transformer les métaphores les plus vives en symboles, et aussi du côté de la réception naturelle du langage, qui elle, en revanche, tend à donner une interprétation métaphorique là où cela est possible. Il est donc tout à fait clair que même si nous sommes d'accord avec Le Guern pour admettre que les différences entre métaphores et symboles sont nettes sur le plan théorique, il n'en est pas du tout ainsi dans la pratique de l'interprète ou du traducteur La distinction entre symbole et métaphore n'est donc pas suffisante pour savoir si la métaphore (au sens large) est traduisible. 
Il me semble que ce qui permet à une métaphore d'être traduite littéralement, c'est avant tout sa «pureté» ou sa «netteté» en tant que figure de pensée. Car, contrairement à ce que pense le Groupe de Liège (1970), la métaphore est bien une figure de pensée. La métaphore-symbole, nous l'avons vu, est une figure de pensée par excellence, tout comme la similitude. La métaphore propre est elle aussi une figure de pensée même si elle est fondée sur la sélection d'un sème, à condition qu'elle ne soit pas morte, figée ou conventionnalisée. Car plus la métaphore est «pure», c'est-à-dire qu'elle n'a pas été «contaminée» par un contexte linguistique, social, culturel ou littéraire, et qu'elle n'a pas été atteinte par le phénomène d'idiome ou de phraséologie, plus son interprétation exige un lourd travail logique et pragmatique. Plus le lexème servant de véhicule (au sens de Richards 1936) aura des connotations propres à un groupe linguistique social ou culturel, plus il sera pur et net en tant que figure de pensée, et approprié à une traduction littérale.

Ainsi la métaphore de Pascal «L'homme n'est qu'un roseau» doit être traduite mot à mot par toute langue qui possède des équivalents pour ces mots. $\mathrm{Ma}$ thèse voulant que toute métaphore pure soit en principe traduisible mot à mot, est basée sur l'hypothèse que les locuteurs de toutes les langues ont à peu près le même savoir encyclopédique, c'est-à-dire que les différences du savoir encyclopédique qu'on trouve parmi les gens parlant différentes langues ne sont pas plus grandes que celles qu'on trouve parmi les gens parlant la même langue. En plus de ce savoir extralinguistique égal, ils ont aussi une égale compétence logique, pragmatique et symbolique. Notre hypothèse est donc que les gens de toutes commnautés linguistiques et culturelles ont à peu près le même savoir sur le monde et les mêmes modes de pensée. Cette hypothèse est nécessaire pour que soit possible toute traduction plus ou moins littérale, sans rapport spécial à la traduction de la métaphore.

Nous savons que pour traduire il faut d'abord comprendre (c'est d'ailleurs la principale raison des difficultés immenses de la traduction automatique: l'ordinateur ne comprend pas). Comprendre, cela veut dire comprendre un énoncé, et non pas une phrase, qui n'est qu'une essence abstraite créant un rapport entre une représentation phonétique et une ou plusieurs représentations sémantiques. L'énoncé, en revanche, est une réalisation de la potentialité de la phrase, qui contient d'habitude seulement un des sens cle la phrase, et qui transmet en plus du sens grammatico-sémantique tout un groupe de sous-entendus qui ne font pas partie de la représentation sémantique (Sperberg 1975). L'énoncé est un rapport entre une représentation phonétique et une représentation conceptuelle qui est déterminée par la grammaire, la sémantique et par un large et complexe input pragmatique, déterminé par le savoir encyclopédique du locuteur et du destinataire, par la situation, les signes extralinguistiques et les énoncés antérieurs. Comprendre un énoncé est donc une chose complètement différente que de comprendre une phrase: pour comprendre un énoncé il nous faut une pensée pragmatique en plus de la pensée logico-sémantique. C'est la pensée pragmatique qui permet au locuteur de dire moins que ce qu'il veut transmettre (le destinataire complétera de lui-même) et aussi de dire une chose avec l'intention d'en dire une autre (par exemple, dans le cas de l'ironie).

Il nous faut donc accepter l'hypothèse que les locuteurs de toutes les langues pensent et parlent à peu près de la même façon, sinon, il n'y aurait pas possibilité de traduction, mais seulement d'interprétation ou d'un mélange des deux. Cela nous mène à dire qu'une expression comme «idée verte» est nécessairement une anomalie sémantique dans toutes les langues du monde, bien que dans telle langue elle puisse être une expression idiomatique régulière, et comme telle faire partie de la compétence linguistique, alors que dans une autre langue elle est une parfaite nouveauté, qui pourra être comprise seulement si le destinataire met en œuvre, non seulement sa compétence linguistique mais aussi sa compétence pragmatique, voire symbolique. La compétence pragmatique, dans ce cas, étant la possibilité d'interpréter les mots «idée verte» comme, par exemple, idée qui n'est pas venue à sa maturité, et la compétence symbolique étant, par exemple, la possibilité de faire de la notion de fruit vert un signifiant de la notion de danger: l'idée qui n'est pas venue à sa maturité est aussi dangereuse pour ses tenants qu'est le fruit vert pour celui qui le mange.

Ce type de raisonnement, qui est à la fois logique, pragmatique et symbolique, se fait chaque fois qu'on se trouve devant une métaphore pure, qu'elle soit métaphore propre ou métaphore-symbole. La nécessité de la part du destinataire de s'engager dans ce type de raisonnement, quand il s'agit, du point de vue théorique, d'une métaphore propre, peut être due au fait que cette métaphore, fondée sur la sélection d'un sème, ne peut pas être comprise comme telle, parce que le sème sélectionné n'appartient pas au lexème employé dans la compétence du destinataire. Mais, comme le dit Le Guem (1980: 68) : «l'absence d'un sème dans le signifié d'un lexème n'implique pas la méconnaissance dans l'objet désigné du trait de substance correspondant : on pourra donc interpréter comme symbole la métaphore dont l'interprétation était bloquée». Une métaphore interprétée comme symbole est une figure de pensée pour le destinataire, et non pas une figure sémantique ou sémique, donc elle est ce que nous avons appelé «métaphore pure».

Il est clair que plus la métaphore est audacieuse, plus libre dans la création et plus unique et originale, plus elle aura de chances d'être une métaphore de pensée et sera plus facilement reconstituée dans une autre langue (Kloepfer 1967: 116), à condition que l'originalité provienne d'un nouveau symbolisme pour le destinataire. Mais si l'originalité est due, par exemple, à un nouvel usage d'une ancienne locution idiomatique, la métaphore, tout en étant maintenant une nouvelle figure de pensée, reste «contaminée», 
entachée d'idiotisme, et il n'y a plus de métaphore pure. L'absence de tout idiotisme, de toute connotation spéciale, linguistique, culturelle, littéraire, de groupes sociaux, etc., est une condition nécessaire pour la métaphore pure, qu'elle soit métaphore propre ou métaphore-symbole.

Ceux qui pensent que la vivacité et l'originalité de la métaphore peuvent être mesurées selon l'impossibilité de la traduire (voir, par exemple, Dagut 1974 ; 1976) parlent en fait de la métaphore «impure». Il est bien possible que ce dernier type de métaphore soit le plus fréquent des deux, non seulement quand il s'agit du stock plus ou moins conventionnel des métaphores en usage dans la presse, mais aussi en ce qui concerne les métaphores non conventionnelles de la litterature et de la poésie. Des deux genres de métaphores «impures», celles de la presse et celles de la littérature, les dernières, il est vrai, sont plus difficiles à traduire, justement en raison de leur vivacité et de leur originalité. Mais il ne faut pas conclure, à partir de cette conséquence empirique, que plus la métaphore est vive et originale plus elle est difficile à traduire, cela n'étant vrai que pour les métaphores de type «impur».

Pour terminer, voici deux exemples de métaphores pures, traduites de l'hébreu moderne:

(1) En route vers les combats

$\mathrm{J}$ 'ai été vaincu

Maintenant je suis étendu dans le sang de mes pensées.

(Shin Tamari, la Montagne de la lumière)

(2) La voici à Londres ... Voici qu'un portail s'ouvre dans un autre jardin.

Des oiseaux gazouillent en anglais.

(Nathan Shaham, Aller et retour)

Il est clair qu'il n'y a aucun lien spécial entre l'hébreu et le français qui puisse permettre cette traduction de mot à mot. D'ailleurs, il n'est pas besoin d'être polyglotte pour savoir si telle métaphore est traduisible: il suffit de se demander s'il est possible de changer le mot métaphorique par un synonyme sans créer par là une bizarrerie. Si cela est possible, nous sommes devant une métaphore pure qui, en principe, est traduisible.

MOSHE AZAR Université de Haïfa, Israël

\section{BIBLIOGRAPHIE}

DAGUT, M.-B. (1974) : «La métaphore comme problème spécial dans la traduction (en hébreu), $\mathrm{Ha}$ sifrut/Literature, 18-19, pp. 120-136.

DAGUT, M.-B. (1976): «Can «Metaphor» Be Translated ?», Babel, 12, pp. 21-23.

GROUPE, M. (1970): Rhétorique générale, Paris, Larousse.

KLOEPFER, R. (1967): Die Theorie der Literarischen Übersetzung, München, Fink.
LE GUERN, M. (1973) : Sémantique de la métaphore et de la métonymie, Paris, Larousse.

LE GUERN, M. (1981): «Métaphore et argumentation», in l'Argumentation, Lyon, Presses universitaires de Lyon, pp. 65-74.

RICHARDS, I.A. (1936) : The Philosophy of Rhetoric, London, Oxford University Press.

SPERBER, D. (1975): «Rudiments de rhétorique cognitive», Poétique, 23, pp. 389-415. 